\title{
ACKNOWLEDGMENTS TO REVIEWERS 2012
}

Herewith the Editorial Committee of the Chemical and Process Engineering quarterly (Inżynieria Chemiczna i Procesowa) expresses our sincere words of gratitude to all peer-reviewers for their concerted efforts at assessment of manuscripts submitted in 2012. The evaluation concerned both the formal requirements as well as scientific merit of the submissions. We are thankful for the honest and invaluable work of all the referees, which highly contributed to the recent growth in the scientific standing and dissemination of our quarterly. The reviewers' names are listed below.

\section{LIST OF REVIEWERS 2012}

Ambrożek Bogdan

Antos Dorota

Badur Janusz

Bałdyga Jerzy

Barigou Mostafa

Berezowski Marek

Biń Andrzej

Bodzek Michał

Borgianni Carlo

Brunazzi Elisabetta

Buczek Bronisław

Burghardt Andrzej

Carlston Andreas

Chavez Torres Rosa Hilda

Ciach Tomasz

Colombani Jean

Corton Eduardo

DeDiego Luis F.

Douglas Timothy

Du Plessis Erika

Evans Geoffrey M.
Gac Jakub

Gierycz Paweł

Głuszcz Paweł

Górak Andrzej

Grzesik Mirosław

Henczka Marek

Hupka Jan

Jandacka Jozef

Jarosz-Wilkołazka Anna

Jun Yue

Kaczmarski Krzysztof

Kalina Jacek

Kamieński Jerzy

Kamiński Władysław

Karcz Joanna

Kawalec-Pietrenko Bożenna

Kiljański Tomasz

Kmieć Andrzej

Kolat Pavel

Kołtuniewicz Andrzej

Konstanciak Anna 
Kordylewski Włodzimierz

Kowalski Stefan J.

Kozaczka Jarosław

Krzystek Liliana

Kucaba-Pietal Anna

Kupiec Krzysztof

Lampke Thomas

Ledakowicz Stanisław

Lisiene Jolanta

Liwarska-Bizukojć Ewa

Lucia Angelo

Łach Jan

Malinowski Janusz

Marijanssen Jan

Mianowski Andrzej

Molga Eugeniusz

Moskal Arkadiusz

Mościcki Lech

Mozgawa Włodzimierz

Nastaj Józef

Orciuch Wojciech

Pakowski Zdzisław

Pamuła Elżbieta

Park So-Jin

Peryt-Stawiarska Sylwia

Piotrowski Krzysztof

Pirożyński Mirosław

Podgórska Wioletta

Poplewski Grzegorz

Porteiro Jacobo

Posmyk Andrzej

Pronobis Marek

Radomiak Henryk

Rakoczy Rafał

Roubinek Otto

Rożeń Antoni
Rudniak Leszek

Rymowicz Waldemar

Sieniutycz Stanisław

Skalska Kinga

Szaniawska Daniela

Sztaba Kazimierz

Szymanek Arkadiusz

Tal-Figiel Barbara

Thullie Jan

Trusek-Hołownia Anna

Urbaniak Włodzimierz

Vienken Joerg

Warmuziński Krzysztof

Werle Sebastian

Wesołowski Piotr

Witczak Stanisław

Witrowa-Rajchert Dorota

Wolicka Dorota

Xiongwen Zhang

Zarzycki Roman

Zen Chen

Zhang Xioalei 